\title{
Propuesta metodológica prospectiva para la elaboración de un índice sintético de vulnerabilidad hidrogeológica: el caso de estudio de Maneadero en México*
}

\author{
Prospective Methodological Proposal for the \\ Elaboration of a Synthetic Index of Hydrological \\ Vulnerability: the Case Study in Maneadero, Mexico
}

\section{Ana Huaico-Malhue**, Alfredo Pérez-Morales ${ }^{* * *}$, Luis W. Daessle**** Recibido: 2017-03-08 // Aprobado: 2017-04-20// Disponible en linea: 2017-06-30}

Cómo citar este artículo: Huaico-Malhue, A., Pérez-Morales, A. y Daessle, L. W. Propuesta metodológica prospectiva para la elaboración de un índice sintético de vulnerabilidad hidrogeológica: el caso de estudio de Maneadero en México. Ambiente y Desarrollo, 21(41), 107-121. https:/doi. org/10.11144/Javeriana.ayd21-41.pmpe doi:10.11144/Javeriana.ayd21-41.pmpe

\begin{abstract}
Resumen
En América Latina existe un serio problema de disponibilidad y de calidad de los datos sobre aspectos tan relevantes como el estado de los acuíferos. Ante esta situación, es urgente la elaboración de metodologías de diagnóstico simplificadas. Se propone un índice de vulnerabilidad hidrogeológica basado en 32 indicadores universales. Luego de la aplicación de técnicas cualitativas multicriterio, el número de indicadores se redujo a un total de tres: uso de la tierra, sólidos disueltos totales y niveles de agua subterránea. Se identificó un área de vulnerabilidad hidrogeológica crítica asociada con el desarrollo urbano. Se concluye que la falta de datos para la aplicación de modelos estandarizados se puede resolver con la selección de indicadores claves mínimos relevantes para cada área de estudio.
\end{abstract}

Palabras clave: hidrogeología; manejo de recursos hídricos; indicadores

\footnotetext{
* Este artículo hace parte del proyecto de investigación La percepción social, la estimación y la medición del riesgo ambiental en la peninsula de Baja California UABC/220, Universidad Autónoma de Baja California.

** Doctora en Medio Ambiente y Desarrollo, académica de la Facultad de Recursos Naturales de la Universidad Católica de Temuco, Chile. Correo electrónico: anitahuaico@gmail.com. Autora de correspondencia.

*** Doctor en Geografía y Académico de la Universidad de Murcia, España. Correo electrónico: alfredop@um.es **** Doctor en Geología/Geoquímica. Investigador y docente de la Universidad Autónoma de Baja California, México. Correo electrónico: walter@uabc.edu.mx
} 


\begin{abstract}
There is a serious problem regarding the availability and quality of data on aspects as important as the status of aquifers in Latin America. Faced with this situation, it is urgent to elaborate simplified diagnostic methodologies. We propose a hydrogeological vulnerability index based on 32 universal indicators. After the application of qualitative multi-criteria techniques, we reduced the number of indicators to a total of three: land use, total dissolved solids, and groundwater levels. We identified an area of critical hydrogeological vulnerability associated with urban development. We conclude that the lack of data for the application of standardized models can be resolved with the selection of minimum key indicators relevant to each study area.
\end{abstract}

Keywords: hydrogeology; water resources management; indicators 


\section{Introducción}

La bibliografía científica refleja la preocupación por la degradación de los acuíferos. En este sentido, Sharma (1998) menciona que los cambios secuenciales en la profundidad de los acuíferos afectan su calidad, y que el decrecimiento de la infiltración en las cuencas debido a la escasez de lluvias causa y acentúa los procesos de erosión producto de la reducción de la cobertura vegetal natural. También, en un estudio realizado en Jordania, Al-Alawi (2008) indica que para satisfacer la creciente demanda de alimentos, se extendieron las siembras a zonas de baja precipitación que hasta entonces no habían sido cultivadas por sus escasas aptitudes agrícolas, dando lugar a la degradación de suelo de manera acelerada. El trabajo señala que el aumento de la inversión en diferentes proyectos de riego por el sector privado supuso un incremento de la superficie cultivada, mediante prácticas intensivas que motivaron la sobreexplotación de recursos hídricos. Esta correlación positiva entre degradación hidrogeológica y agricultura ha sido también identificada en otros lugares en el mundo, tal y como lo demuestra el trabajo realizado por Saiko y Zonn (2000) en la región de Circum-Aral (Priaraliye en Rusia).

Los cambios que pueden experimentar los sistemas hídricos en climas sensibles a las variaciones del recurso natural vienen explicados, en gran medida, por los desequilibrios que se producen en las relaciones hombre-medio. En estos términos, las conexiones y vínculos que se establecen entre el medio humano y el natural representan elementos de vital importancia para salvaguardar el equilibrio ambiental. Se trata de un planteamiento antropocéntrico que explica la estabilidad de estos sistemas en función de la capacidad de adaptación de la sociedad que hace uso de este. Por tanto, el éxito en la relación depende, en primer lugar, de la actividad del hombre y su capacidad de adaptación y, en última instancia, la vulnerabilidad biofísica del sistema.

En lo que respecta a la vulnerabilidad biofísica, actualmente parece consensuado que se trata de un término frecuentemente relacionado con la exposición y la sensibilidad de aquello que se preste a esa condición (Eakin y Luers, 2006; Gallopin, 2006; Yohe y Tol, 2002). El primero, exposición, se puede definir como los elementos que son susceptibles de ser afectados por una amenaza o peligro, es decir, en el caso que nos ocupa, el área de influencia de un acuífero quedaría expuesta al peligro por la sobreexplotación de los recursos hídricos, en este caso el suelo. Todo lo anterior suele venir representado por indicadores que nos facilitan la evaluación de la vulnerabilidad.

Un indicador o conjunto de ellos integrados en un índice, es una herramienta que adquiere una utilidad para: (a) detectar áreas frágiles o propensas a sufrir degradación, (b) trazar las áreas donde la degradación ya es un problema, (c) estimar su gravedad o (d) comprender mejor los procesos asociados con la degradación. En definitiva, nos permiten evaluar y delimitar espacialmente la vulnerabilidad en la que se encuentra un ambiente.

Pese a las bondades en el uso de los indicadores, en ocasiones pueden ser redundantes para reflejar una realidad, o no hay disponibilidad de los datos, por lo que pensamos que las zonas que están teniendo síntomas de degradación hídrica y que no cuentan con todos los datos para realizar una evaluación con parámetros y modelos de precisión avanzados, no deben quedar al margen de las evaluaciones que permitan obtener una aproximación acerca de su estado, como es el caso de la gestión de los recursos hídricos.

En este contexto, es muy importante experimentar con ellos para seleccionar un conjunto mínimo que proporcione la máxima información sobre cambios, tendencias y calidad de los recursos. Para solventar esa deficiencia, en otros estudios hidrológicos ha sido aplicado el análisis multicriterio de forma satisfactoria (Garfi et al., 2013; Chowdhury y Rahman, 2008; Valle Junior et al., 2015), ya que sirve para proporcionarnos la idea de los indicadores verdaderamente significativos. Se trata de una depuración muy necesaria, ya no solo en lo que respecta al problema de la perturbación de carácter estadístico, sino también para la cuestión de la pobreza de datos e información que existe en países menos desarrollados, lo que supone una oportunidad y punto de partida para aplicar este tipo de trabajos en ámbitos espaciotemporales con mayor resolución. 
Por todo lo anterior, este trabajo tiene por objetivo buscar los indicadores hidrológicos mínimos que permitan caracterizar de una manera prospectiva la vulnerabilidad hidrogeológica mediante un índice sintético aplicado en un acuífero a escala local en la localidad de Maneadero, en la península de Baja California al noroeste de México.

El trabajo utiliza el método deductivo, ya que una vez establecido un conjunto de indicadores hidrológicos universales en desertificación, se seleccionan los pertinentes entre los disponibles. Se piensa que el uso de esta metodología puede ser replicable para elaborar índices en otras áreas con otras particularidades, con escasez de datos, falta de datos correlativos y deficiente calidad de la información disponible.

\section{Área de estudio}

El acuífero costero de Maneadero se encuentra ubicado en el municipio de Ensenada, en el estado de Baja California al noroeste de México, entre los meridianos $116^{\circ} 30^{\prime}$ y $116^{\circ} 40^{\prime}$ de longitud oeste y los paralelos $31^{\circ} 41^{\prime}$ y $31^{\circ} 51^{\prime}$ de latitud norte, y climáticamente emplazado en una zona mediterránea (figura 1). Con respecto a su recarga, recibe volúmenes de agua procedentes de los escurrimientos que concurren al sitio, siendo los principales los de los arroyos de San Carlos y Las Ánimas. La alimentación vertical que complementa a la anterior llega de las precipitaciones extraordinarias que ocurren en la cuenca vertiente y a la recarga por infiltración a través de la escorrentía concentrada de mediano tamaño sobre la superficie permeable de la cuenca.

Los análisis se realizaron sobre una superficie de 6043 hectáreas, que corresponde al área superficial del acuífero de Maneadero y que incluyen las 4200 hectáreas parceladas de uso agrícola que presentaba para el año 2010 (OEIDRUS, 2010). La localidad posee una población de 30656 habitantes (INEGI, 2010). De acuerdo con OEIDRUS (2014), "El poblado de Maneadero se ha consolidado como una de las regiones agrícolas más importantes de Baja California y la República Mexicana, por su alta producción de hortalizas y la calidad de sus cultivos". "Actualmente, en esas tierras viven alrededor de 200 ejidatarios con sus familiares, mismos que han constituido una comunidad agrícola".

La importancia de este ámbito hidrogeológico se relaciona directamente con el desarrollo agrícola con vocación de mercado, ya que la mayor parte de su producción se exporta a los Estados Unidos y representa la principal fuente de ingresos de los habitantes del área de influencia del acuífero, a la vez que abastece el $38 \%$ de las necesidades de agua potable de la ciudad de Ensenada (CNA, 2002). Sin embargo, varios problemas están poniendo en tela de juicio la continuidad en el uso de este recurso hídrico subterráneo: los procesos de intrusión marina y la salinización de suelos, unidos a la disminución de los niveles piezométricos y de su calidad, son consecuencias ecológicas y socioeconómicas producto del impacto de estos procesos, tal vez irreversibles, sobre el suelo.

El aprovechamiento del agua subterránea del valle de Maneadero inició desde 1910, sin embargo, las primeras obras importantes con esta finalidad se llevaron a cabo por medio de perforaciones y

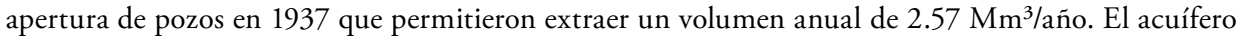
fue vedado desde el 21 de febrero de 1961, debido a que la intensa explotación para fines agrícolas empezó a dar signos de desajuste ambiental, y pese a las medidas de control establecidas, a principios de los setenta el acuífero seguía agravando el desequilibrio entre la recarga y la extracción de agua (SRH, 1971).

$5 \quad$ En México, zona de veda, según la Ley de Aguas Nacionales, se define como: "Aquellas áreas específicas de las regiones hidrológicas, cuencas hidrológicas o acuíferos, en las cuales no se autorizan aprovechamientos de agua adicionales a los establecidos legalmente y éstos se controlan mediante reglamentos específicos, en virtud del deterioro del agua en cantidad o calidad, por la afectación a la sustentabilidad hidrológica, o por el daño a cuerpos de agua superficiales o subterráneos" (Ley de Aguas Nacionales, publicada en el Diario Oficial de la Federación el 1. ${ }^{\circ}$ de diciembre de 1992. Texto vigente. Última reforma publicada DOF 24-03-2016). 


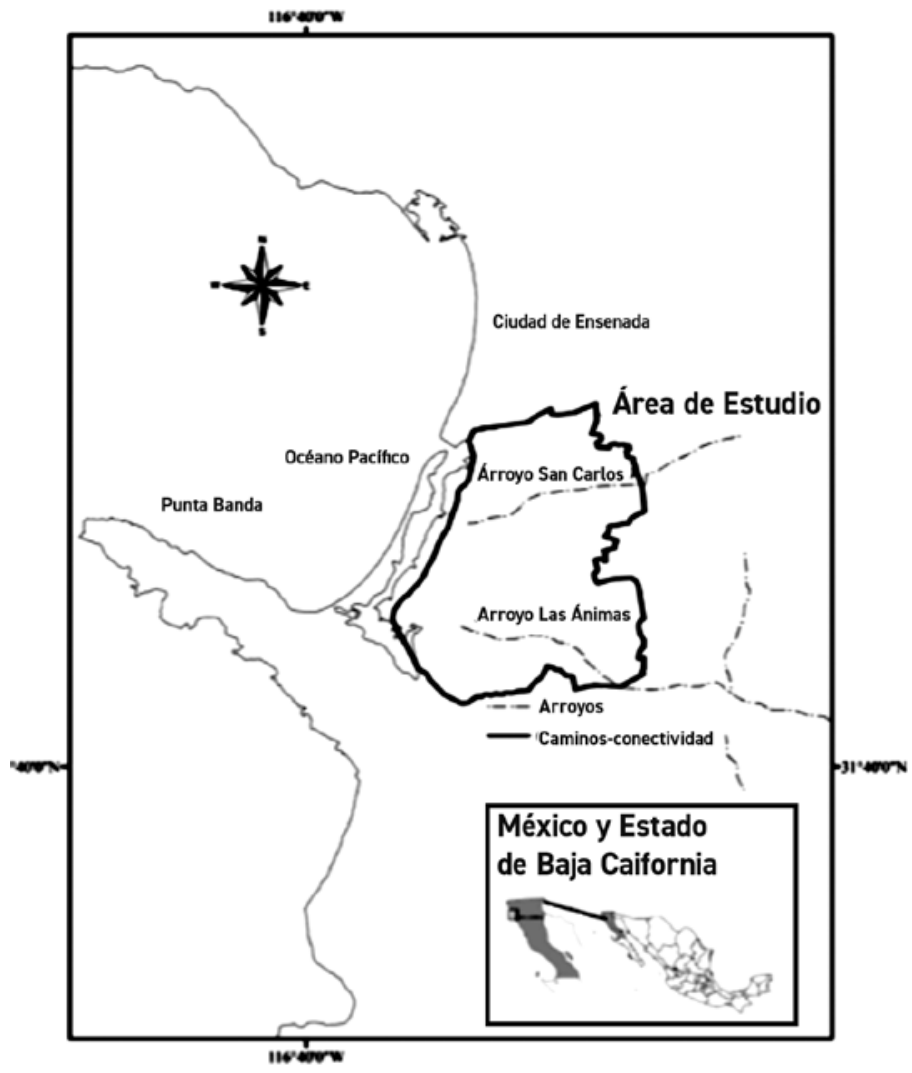

Figura 1. Ubicación del área de estudio

Fuente: elaboración propia.

En el año 2002 se realizó una evaluación acerca de la disponibilidad de los recursos hídricos contenidos en el reservorio natural subterráneo, y se concluyó que la descarga superaba a la recarga en un $23.8 \%$. Por esta razón, el acuífero pasó a ser inmediatamente calificado como "sobreexplotado" (Conagua, 2002).

En la actualidad, los usos de esa agua subterránea siguen siendo la actividad agrícola (67\%), fuertemente dependiente, y los consuetudinarios urbanos (29\%), los cuales, aunque también importantes, obviamente, prevalecen los primeros en el valle de Maneadero, por ser una zona agrícola de alto rendimiento (Licona, 2011).

En la zona se aprecian procesos de intrusión marina que han afectado la salinidad del agua subterránea y con ello su calidad (Daesslé et al., 2014). Por otro lado, existen sectores en los cuales los niveles piezométricos se han profundizado, por lo que se estiman consecuencias ecológicas y socioeconómicas como producto del impacto de estos procesos sobre el suelo. 


\section{Metodología para la aplicación del índice hidrogeológico}

\section{Revisión bibliográfica de indicadores}

La metodología general aplicada consiste en una serie de cuatro pasos secuenciales que llevan a término la realización del índice. Se parte con la revisión bibliográfica de indicadores, seguida por la identificación de la problemática (causas y efectos de la degradación del acuífero en el área de estudio), luego la definición de criterios de selección, para finalmente llevar a cabo la elaboración del índice y su validación mediante contraste de los resultados con la realidad (figura 2). Lo anterior se basó en la revisión de distintas propuestas metodológicas para elaborar índices, especialmente aquella planteada por un trabajo llevado a cabo entre la Organización para la Cooperación y el Desarrollo Económico (OECD) y la Unión Europea, quienes proponen diez pasos para la construcción de indicadores compuestos (OECD/EU/JRC, 2008), que van desde el marco teórico (que en este caso en estudio consistió en la revisión bibliográfica de 170 estudios de casos), hasta la visualización de los resultados (correspondiente a las cartografías de vulnerabilidad).

En el mismo contexto, existen numerosos estudios que han aplicado índices para evaluar la sensibilidad, la vulnerabilidad o la calidad de los acuíferos utilizando sistemas de información geográfica (SIG) para la identificación de zonas, por lo que se puede mencionar especialmente el trabajo de Kardan et al., (2017), quienes realizan el ejercicio de comparar entre el índice DRASTIC y el modelo GALDIT, o el trabajo realizado por Trabelsi et al. (2016), quienes evalúan la vulnerabilidad en un acuífero costero en Túnez. Los modelos con todos los índices estandarizados planteados por estas metodologías no han podido ser aplicadas al área de estudio por la falta de datos, sin embargo, sirvieron como fuente para la elaboración de la propuesta metodológica prospectiva.

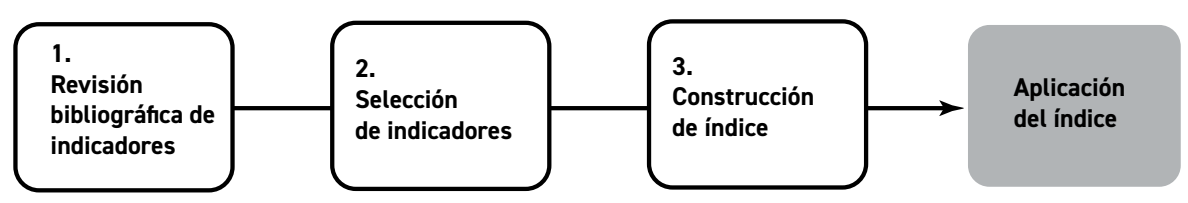

Figura 2. Diagrama metodológico general

Fuente: elaboración propia.

La revisión sobre causas y efectos de la degradación consistió en la selección y el análisis de documentos utilizando criterios establecidos por Berrang-Ford et al. (2011) y por Aldunce et al. (2014). Este método facilita el resumen y la evaluación de los conocimientos actuales sobre un tema determinado (Berrang-Ford et al., 2011; Aldunce et al., 2014).

Para la búsqueda bibliográfica de los anteriores trabajos se accedió a las bases de datos de Springer, Elsevier, Ebsco, Emerald, Wiley y Annual Reviews, empleando como descriptor la palabra clave en el título con las siguientes combinaciones: "hydrological and indicators"; "hydrological and vulnerability"; "aquifer and risk"; "hydrological and assessment"; "aquifer and vulnerability" y "hydrological and monitoring". El periodo de prospección fue desde marzo del año 2011 hasta diciembre del 2015. Como resultado de lo anterior se identificaron un total de 32 indicadores relacionados con la hidrología superficial y subterránea, en una revisión de un total de 170 estudios de casos relacionados con la temática (tabla 1). 


\section{Selección de indicadores}

El análisis previo para la selección de indicadores disponibles y precisos en el área de estudio se realizó siguiendo las orientaciones contenidas en estudios previos sobre la degradación hidrogeológica del área estudio: informes oficiales desde 1978 y publicaciones científicas que reportan observaciones desde el año 2000 (Daesslé et al., 2005, 2009, 2014).

El primer criterio para seleccionar los indicadores fue la pertinencia, es decir, se escogieron aquellos relacionados con las causas y efectos de la degradación hídrica en el área de estudio. El segundo la disponibilidad del dato. El tercero, la disponibilidad por ajuste con la escala de trabajo. Por ejemplo, el indicador de precipitaciones (32) tuvo que ser descartado, porque no estaba disponible en la escala local. Y el cuarto y último fue que el origen de los datos fuera confiable.

Tabla 1. Indicadores utilizados en los 170 casos de estudios

\section{Listado de indicadores hidrológicos}

$1 \quad$ Nivel piezométrico

2 Sólidos totales disueltos

3 Conductividad eléctrica

4 Salinización del agua

5 Sobreexplotación del acuífero

6 Extracción

$7 \quad$ Escasez de agua

$8 \quad$ Calidad del agua

9 Número de bombas de extracción

10 Diversificación en la gestión del uso agua

11 Eficiencia en el uso

12

Cambios en la profundidad de agua subterránea

13 Regulación hidrológica superficial

14 Área irrigada

15 Intensidad de irrigación

16 Intrusión marina
17 Potencial de riego

18 Almacenamiento de agua por escorrentía

19 Consumo de agua por sector

20 Fuga de agua

21 Reciclaje de aguas residuales

22 Disponibilidad de agua

23 Cambios en la red de drenaje

24 Consumo en riego

25 Densidad de drenaje

26 Mineralización y salinización

27 Carga de sedimentos

28 Presencia de embalses

29 Cuerpos de aguas

30 Importación del agua

31 Uso de suelo (indicador indirecto)

32 Precipitaciones

Fuente: elaboración propia. 


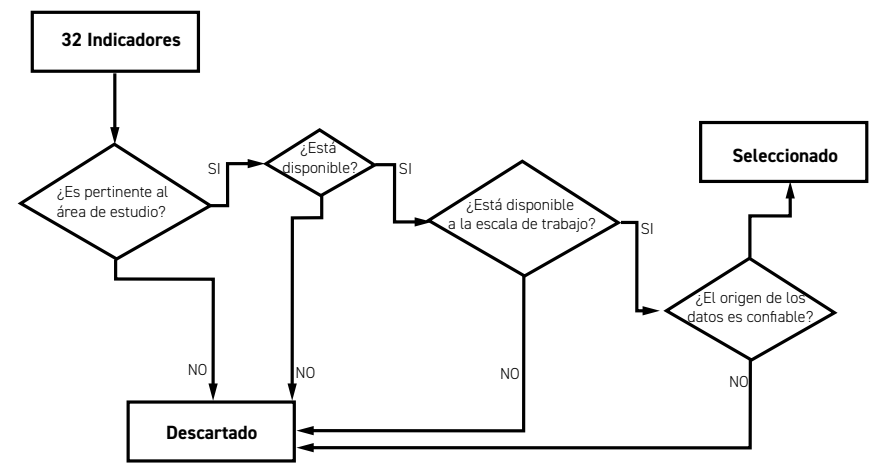

Figura 3. Diagrama de flujo para la selección de indicadores

Fuente: elaboración propia.

Como resultado de la aplicación de este procedimiento de selección (figura 3) se marginaron los siguientes indicadores: uso de suelo, sólidos totales disueltos (STD) y niveles piezométricos (tabla 2).

Tabla 2. Datos y origen de las fuentes

\begin{tabular}{lll}
\hline \multicolumn{1}{c}{ Dato } & \multicolumn{1}{c}{ Fuente } & \multicolumn{1}{c}{ Año } \\
\hline Niveles piezométricos & Manjarrez & 2011 \\
\hline Sólidos totales disueltos & Manjarrez & 2011 \\
\hline Uso de suelo & Fotografías aéreas & 2011 (INEGI) \\
\hline
\end{tabular}

Fuente: elaboración propia.

\section{Construcción del índice de vulnerabilidad hidrogeológica}

Para la realización del índice hubo que componer la información espacialmente, mediante la interpolación de 45 puntos de muestreo tomados por Manjarrez (2011) de manera aleatoria de los indicadores hídricos (figura 4). Se seleccionó el método Kriging, debido a que estudios como el realizado por Esteller et al. (2005) mencionan que procedimientos similares presentan un error de estimación mayor para el mapeo de acuíferos. Los diferentes usos de suelos fueron identificados a través de fotointerpretación.

Como paso previo al trazado de la cartografía, se llevó a cabo una tabla de correspondencia para la reclasificación de los indicadores y asignación de valores según unos umbrales preestablecidos (tabla 4). Para la precisión de estos en cada uno de los indicadores se siguieron las pautas contenidas en algunas de las publicaciones consultadas en el paso de la revisión bibliográfica:

- Uso del suelo (Conagua, 2014): a aquella actividad con mayor consumo de agua se le asigna el peso más alto.

- Sólidos disueltos totales: según los parámetros establecidos por la Norma Oficial Mexicana: NOM127-SSA1-1994 (SSA, 2001), que regula los límites permisibles para uso humano, y FAO (1985), que plantea las directrices y grados de restricción para uso agrícola.

- Los niveles piezométricos: cuyos pesos fueron adaptados de la metodología DRASTIC (Aller et al., 1987). 


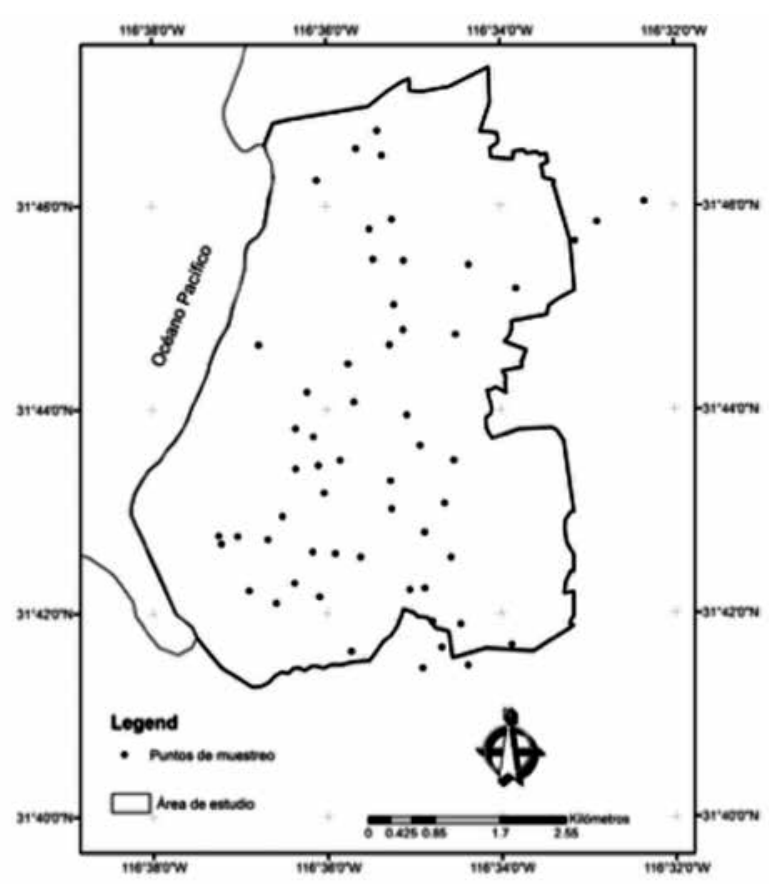

Figura 4. Localización de los puntos de muestreo

(sistema de coordenadas geográficas WGS84)

Fuente: Manjarrez (2011).

Tabla 4. Ponderadores para los diferentes indicadores

Criterios de calidad

\begin{tabular}{llll}
\hline \multicolumn{1}{c}{ Indicador } & \multicolumn{1}{c}{ Alta } & \multicolumn{1}{c}{ Media } & \multicolumn{1}{c}{ Baja } \\
\hline $\begin{array}{l}\text { Sólidos totales disuel- } \\
\text { tos }(\mathrm{mg} / \mathrm{L})\end{array}$ & $\begin{array}{l}\text { Hasta } 1000 \\
\text { (permisible al uso } \\
\text { humano) }\end{array}$ & $\begin{array}{l}\text { 1001-2000 } \\
\text { (con restricción agríco- } \\
\text { la de moderada a leve) }\end{array}$ & $\begin{array}{l}\text { 2000 en adelante } \\
\text { (con restricción agrí- } \\
\text { cola severa) }\end{array}$ \\
\hline Nivel piezométrico & 30 metros & $30.1-50$ metros & 50.1 metros y más \\
\hline Uso de suelo & $\begin{array}{l}\text { Matorral arborescen- } \\
\text { te, de suculentas y } \\
\text { vegetación nativa }\end{array}$ & $\begin{array}{l}\text { Urbano y medio } \\
\text { construido }\end{array}$ & $\begin{array}{l}\text { Cultivos agrícolas } \\
\text { anuales }\end{array}$ \\
\hline Peso & 1 & 1.6 & 2 \\
\hline
\end{tabular}

Fuente: elaboración propia, basada en los indicadores de sensibilidad ambiental (Kosmas et al., 1999). 
Una vez asignados los valores o pesos a cada unidad del territorio por cada indicador, se utilizó como herramienta para integrar los indicadores el Sistema de Información Geográfica, con el que fueron clasificados y procesados utilizando el logaritmo (ecuación 1). El procedimiento anterior también fue utilizado en los trabajos de Kosmas (1999) y Ochola y Kerkides (2004), para evaluar procesos de desertificación. Además, el mismo principio lo usaron trabajos que utilizan indicadores hidrogeológicos: Farajzadeh y Nik Egbal (2007) y Bakr et al. (2012).

$$
\left(\mathrm{STD}^{*} \text { nivel piezométrico * uso de suelo) }{ }^{1 / 3} \quad \text { (ecuación } 1\right)
$$

Posteriormente, se realizó un análisis exploratorio del conjunto de los datos para llevar a cabo un análisis de conglomerados clasificando los datos en $\mathrm{K}$ medias, un procedimiento de análisis de conglomerados que permite agrupar datos, lo que posibilitó asimismo definir cuatro clases de vulnerabilidad: baja, media, alta vulnerabilidad y zonas críticas (tabla 5).

Tabla 5. Tipología de vulnerabilidad hidrogeológica

\begin{tabular}{ll}
\cline { 2 - 2 } Clases & \multicolumn{1}{c}{ Tipo } \\
\cline { 2 - 2 } $0.00-1.21$ & Baja vulnerabilidad \\
\hline $1.22-1.45$ & Mediana vulnerabilidad \\
\hline $1.46-1.85$ & Alta vulnerabilidad \\
\hline $1.86-+$ & Crítica \\
\hline
\end{tabular}

Fuente: elaboración propia.

\section{Resultados}

\section{Análisis espacial de los indicadores considerados en el índice}

Con respecto al nivel piezométrico, se obtuvo como resultado que un $57.1 \%$ del área del acuífero se encuentra a nivel superficial, es decir, a menos de 30 metros de profundidad (calidad alta), esto se debe a su condición de acuífero costero. Los valores de calidad baja (es decir, que los niveles piezométricos superan los 50 metros) se encuentran en el sector intermedio del área de estudio y representan un porcentaje del $42.9 \%$ de toda la superficie, lo que da cuenta del sector en que los niveles piezométricos han sido más afectados por la extracción de agua (figura 4).

En cuanto a los sólidos disueltos totales, la información proveniente de la fuente (puntos de muestreo) proporciona una medida cualitativa de la cantidad de iones disueltos en el agua. En Maneadero, el $99 \%$ del área presenta una baja calidad, lo que según la Norma Oficial Mexicana se traduce en una restricción agrícola severa.

El uso de suelo relacionado con el gasto de agua según actividad económica revela que el $56 \%$ del área tiene uso agrícola, lo que conlleva una alta utilización del recurso. Esto es relevante, ya que el área ha tenido este uso de suelo histórico, lo que probablemente sea la causa principal del proceso de degradación.

\section{Zonificación de áreas vulnerables hidrogeológicamente mediante la} aplicación del índice sintético

Tras la aplicación espacial del índice se pudo identificar y clasificar tres zonas con diferentes niveles de vulnerabilidad o desarrollo en su proceso de degradación. En el ámbito geográfico del acuífero, el área con una baja vulnerabilidad corresponde a una superficie de 223 hectáreas, ubicadas principalmente en un área focalizada en las riberas del arroyo Las Ánimas. El indicador principal que 
determina esta categorización es el nivel piezométrico, ya que en este sector las alturas se encuentran cercanas a la superficie, debido en gran medida a la influencia del nivel medio del mar. Los otros dos parámetros (uso de suelo y TSD) cuentan con valores de baja calidad. Lo anterior es relevante, ya que se interpreta una disponibilidad a nivel superficial de agua, pero cuya calidad es baja en un sector con alta demanda del recurso.
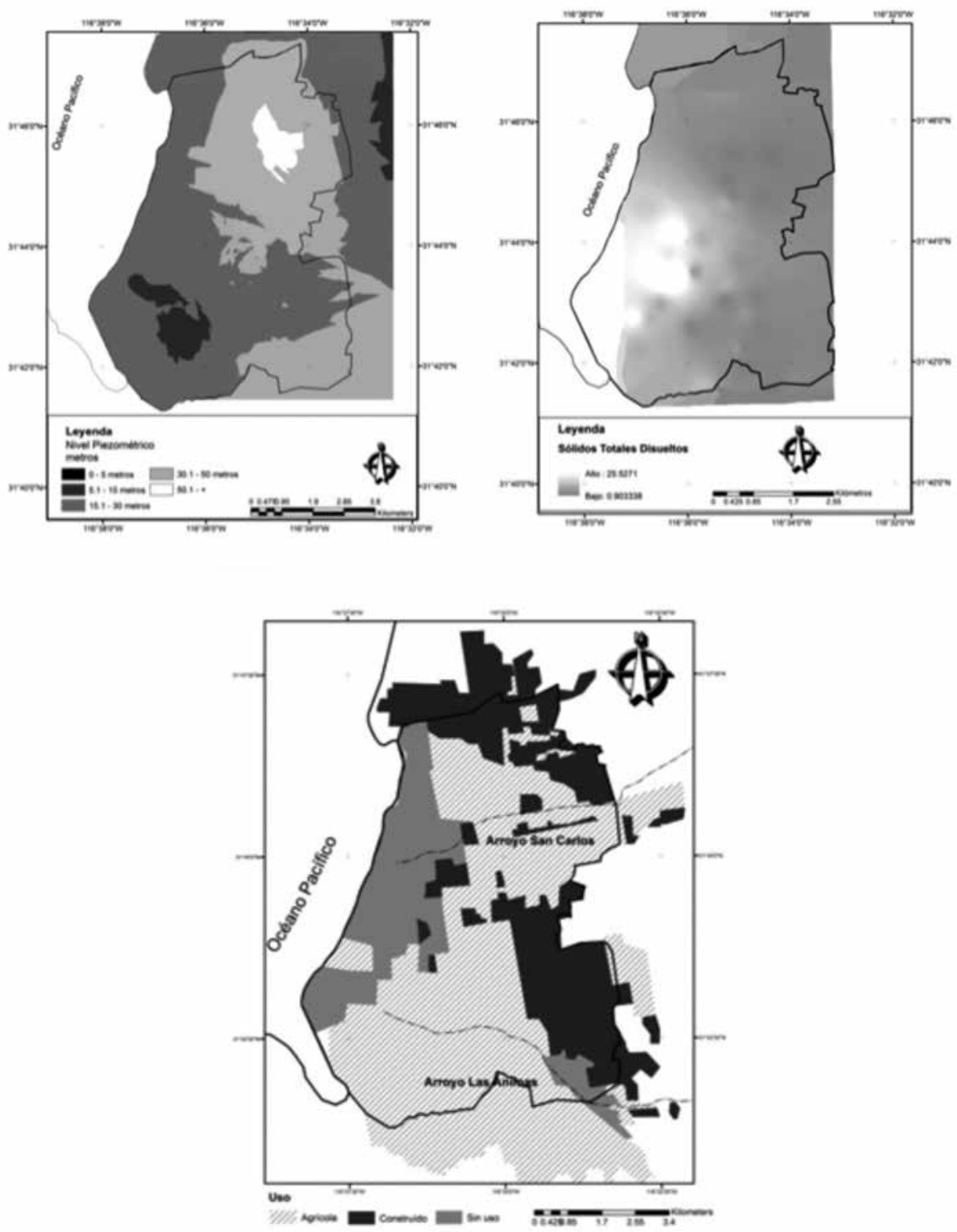

Figura 5. Mapas temáticos por indicador

Fuente: elaboración propia. 
Un segundo fragmento, de 1818 hectáreas, que también se ubica en torno al arroyo Las Ánimas, es el área que se distribuye desde la zona intermedia del afluente a la desembocadura en el mar. Este lugar presenta niveles piezométricos a mayor profundidad, por lo que posee un nivel de vulnerabilidad catalogada como medio.

La zona clasificada como de alta vulnerabilidad por el índice elaborado, se localiza en su mayor parte en las inmediaciones del arroyo San Carlos y al este del afluente de Las Ánimas. Su superficie alcanza unas 3174 hectáreas, lo que equivale a un $52.5 \%$ del total del área de estudio. Se trata de terrazgos con fines agrícolas, baja calidad del agua y niveles piezométricos profundos.

Por último, los resultados cartográficos permitieron delimitar un sector al norte de Maneadero con índices críticos o valores extremos de vulnerabilidad. El uso generalizado de esta área es urbano, pues es donde se ubican los principales núcleos de población. Se interpreta que la presión sobre el acuífero en cuanto a su explotación es máxima y muy concentrada en dicho lugar, lo que ocasiona unos niveles piezométricos profundos que, prácticamente, limitan y restringen de forma severa a la agricultura, por lo que se le ha dado un uso urbano (tabla 6).

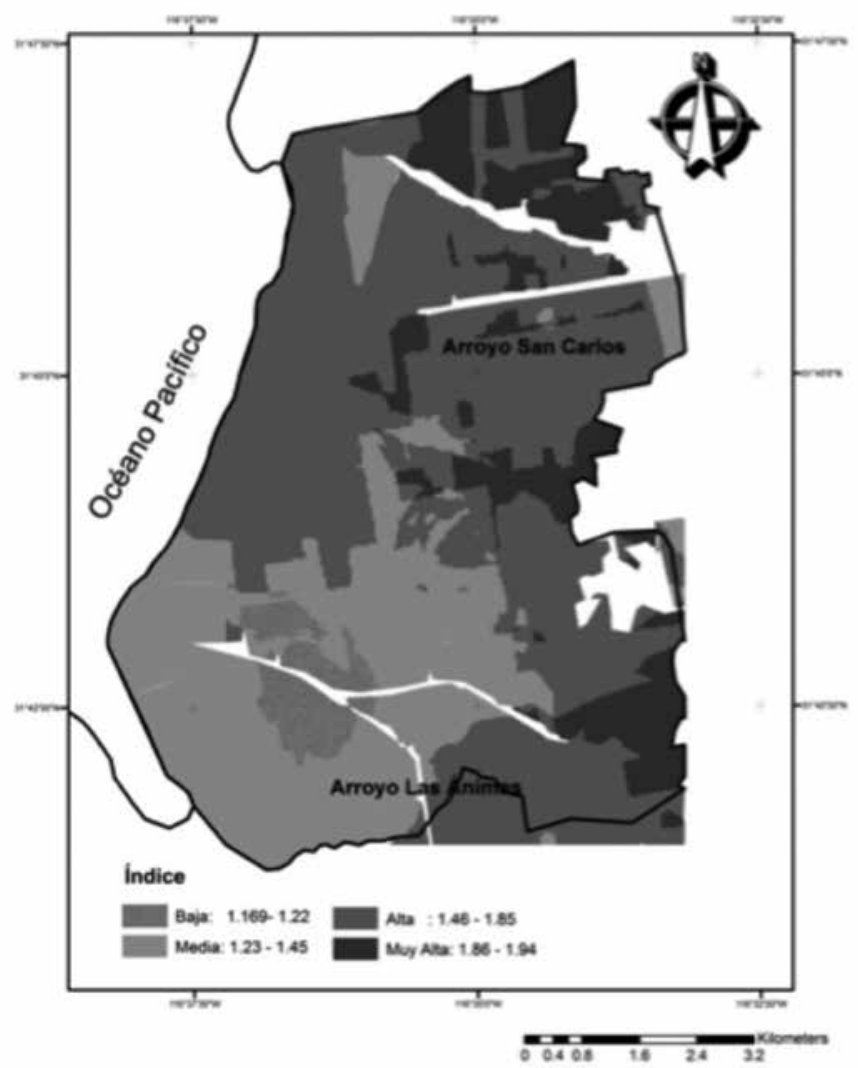

Figura 6. Índice de vulnerabilidad hidrogeológica en Maneadero

Fuente: elaboración propia. 
Tabla 6. Superficie según vulnerabilidad

\begin{tabular}{lccccc}
\hline \multicolumn{7}{c}{ Indice de vulnerabilidad hidrogeológica } \\
\hline \multirow{2}{*}{ Hectáreas } & 223 & Medio & Alta & Critica & Total \\
Porcentaje & 3.7 & 30.1 & 3174 & 828 & 6043 \\
\hline
\end{tabular}

\section{Conclusiones}

El proceso metodológico seguido para la consecución del índice sintético de evaluación de vulnerabilidad hidrogeológica nos lleva a las siguientes conclusiones. En primer lugar, el procedimiento de revisión bibliográfica en repositorios de primer nivel científico facilita la identificación, obtención y selección de los indicadores necesarios, por criterios de escala y confiabilidad para la elaboración de un índice. Gracias a lo anterior, y a la transformación de esas variables mediante unos criterios rigurosos tipificados en cuanto a sus umbrales de clasificación por los trabajos antes consultados, se pudo aplicar dicho índice con un alto grado de éxito en cuanto a la identificación de los sectores sensibles y críticos, ya que la zonificación obtenida se correlaciona con el paisaje y la evidencia de suelos salinos. Desafortunadamente, esa validación que permitiría aseverar con total seguridad el buen ajuste y la bondad del índice no es posible, debido a la falta de más información en el área de estudio. Pese a ello, no se debe desmerecer el resultado final, puesto que supone una primera y valiosa aproximación, además de una herramienta útil y replicable en otros países con características como las del área de estudio, donde la información es ausente en la mayoría de las ocasiones.

En lo que se refiere a los resultados propiamente dichos, permite realizar las siguientes reflexiones interpretativas. La alta vulnerabilidad hidrogeológica que predomina en más de la mitad del acuífero, traducida en una baja calidad hídrica, tiene efectos visibles en toda el área de estudio, con mayor o menor incidencia, ya que el agua de riego elevada del subsuelo posee una alta salinidad, lo que afecta directamente la calidad de los suelos destinados a la agricultura, evidenciando procesos de desertificación y en algunos casos abandono.

Asimismo, la metodología seguida permitió identificar un sector crítico en, que se conjugan los siguientes factores: uso de suelo urbano o construido, niveles piezométricos profundos y baja calidad del agua, lo que hace pensar que la baja calidad hídrica del sector está llevando al abandono de suelos agrícolas para dar paso a la urbanización y con ello a la impermeabilización del suelo, lo que obliga de forma emergente a planificar el área de estudio en su totalidad para identificar zonas de recarga que garanticen la infiltración de agua al acuífero producto de las precipitaciones.

\section{Referencias}

Al-Alawi, M. T. (2008). Desertification in Jordan: A security issue. En Environmental change and human security: recognizing and acting on hazard impacts (pp. 81-102). Springer Netherlands.

Aldunce, P., Indvik, K., Borquez, R., Adler, C. y Galaz, V. (2014). Resilience in the context of climate change: a systematic review of the literature to aid a navigation of diversity. Group of Social Studies-Human Dimension Center for Climate and Resilience Research-CR2 working paper.

Aller, L., Bennett, T., Lehr, J. H. Petty, R. J. y Hackett, G. (1987). Drastic: A standardized system for evaluating ground water pollution potential using hydrogeologic settings. US Environmental Protection Agency, EPA/600/2-87/036. 
Bakr, N., Weindorf, D. C., Bahnassy, M. H. y El-Badawi, M. M. (2012). Multi-temporal assessment of land sensitivity to desertification in a fragile agro-ecosystem: Environmental indicators. Ecological Indicators, 15(1), 271-280.

Berrang-Ford, L., Ford, J. D. y Paterson, J. (2011). Are we adapting to climate change? Global Environmental Change, 21(1), 25-33.

Chowdhury, R. K. y Rahman, R. (2008). Multicriteria decision analysis in water resources management: the malnichara channel improvement. International Journal of Environmental Science Technology, 5(2), 195-204.

Comision Nacional del Agua (Conagua). (2002). Determinación de la disponibilidad del agua en el acuifero de Maneadero, estado de Baja California. Ciudad de México: Conagua, Gerencia de Aguas Subteráaneas, Subdirección General Técnica, Subgerencia de Evaluación y Modelación Hidrogeológica.

Comisión Nacional del Agua (Conagua). (2014). Estadísticas del agua en México. Recuperado de http://www. conagua.gob.mx/CONAGUA07/Publicaciones/Publicaciones/EAM2014.pdf

Daesslé, L. W., Sánchez, E. C., Camacho-Ibar, V. F., Mendoza-Espinosa, L. G., Carriquiry, J. D., Macías, V. A. y Castro, P. G. (2005). Geochemical evolution of groundwater in the Maneadero coastal aquifer during a dry year in Baja California, Mexico. Hydrogeology Journal, 13(4), 584-595.

Daesslé, L. W, Ruiz-Montoya, L., Chandrajith, R., Tobschall, H. J., Camacho-Ibar, V. F., Mendoza-Espinosa, L.G., Quintanilla-Montoya, A. L. y Lugo-Ibarra, K. C. (2009). Fluoride, nitrate and water hardness in groundwater supplied to the rural communities of Ensenada County, Baja California, Mexico. Environmental Geology, (58), 419-429.

Daesslé, L. W., Pérez-Flores, M. A., Serrano-Ortiz, J., Mendoza-Espinosa, L., Manjarrez-Masuda, E., LugoIbarra, K. C. y Gómez-Treviño, E. (2014). A geochemical and 3D-geometry geophysical survey to assess artificial groundwater recharge potential in the Pacific coast of Baja California, Mexico. Environmental Earth Sciences, 71(8), 3477-3490.

Eakin, H. y Luers, A. (2006). Assessing the vulnerability of social-environmental systems. Annual Review of Environment and Resources, (31), 365-394.

Esteller, M. G., Quentin, E. y Díaz Delgado, C. (2005). Uso de sistemas de información geográfica (SIG) para la determinación de parámetros utilizados en la construcción de mapas de vulnerabilidad de acuíferos. Revista Latino Americana de Hidrogeología, 2(1), 17-30.

Food and Agriculture Organization (FAO). (1985). Water quality for agriculture. Recuperado de http://www.fao. org/docrep/003/T0234E/T0234E00.htm\#TOC

Farajzadeh, M. y Nik Egbal, M. (2007). Evaluation of MEDALUS model for desertification hazard zonation using GIS; Study area: Iyzad Khast Plain, Iran. Pakistan Journal of Biological Science, 10(16), 2622-2630.

Gallopin, G. C. (2006). Linkages between vulnerability, resilience, and adaptive capacity. Global Environmental Change, 16(3), 293-303.

Garfi, M., Ferrer-Martí, L., Bonoli, A. y Tondelli, S. (2011). Multi-criteria analysis for improving strategic environmental assessment of water programmes. A case study in semi-arid region of Brazil. Journal of Environmental Management, 92(3), 665-675.

Instituto Nacional de Estadística y Geografía (Inegi). (2010). Censo de población y vivienda. Gobierno de México. Kardan Moghaddam, H., Jafari, F. y Javadi, S. (2017). Vulnerability evaluation of a coastal aquifer via GALDIT 
model and comparison with DRASTIC index using quality parameters. Hydrological Sciences Journal, 62(1), 137-146.

Kosmas, C., Kirkby, M. J. y Geeson, N. (Eds.). (1999). The Medalus Project: Mediterranean desertification and land use: Manual on key indicators of desertification and mapping environmentally sensitive areas to desertification. Directorate-General Science, Research and Development.

Licona, A. (2011). Sistema de información integral del acuifero de Maneadero, B.C., México con fines de Manejo (tesis de maestría en Ciencias en Manejo de Ecosistemas de Zonas Áridas). Universidad Autónoma de Baja California, México.

Manjarrez, E. (2011). Actualización y evolución histórica del avance de la intrusión salina, la contaminación por nitratos y flúor en el acuifero costero de Maneadero, Baja California, México (tesis de licenciatura). Universidad de Guanajuato, Mexico.

Ochola, W. y Kerkides, P. (2004). An integrated indicator-based spatial decision support system for land quality assessment in Kenya. Computers and Electronics in Agriculture, (45) 3-26.

Oficina Estatal de Información para el Desarrollo Rural Sustentable (OEIDRUS). (2010). Panorama general del Valle de Maneadero. Recuperado de http://www.oeidrus-bc.gob.mx/oeidrus_bca/

Oficina Estatal de Información para el Desarrollo Rural Sustentable (OEIDRUS). (2014). Panorama general de Maneadero, Baja California. Recuperado de http://www.oeidrus-bc.gob.mx/oeidrus_bca/pdf/ biblioteca/panoramas/PANORAMA\%20MANEADERO\%202014.pdf

Organización para la Cooperación y el Desarrollo Económicos (OECD)/EU/JRC. (2008). Handbook on constructing composite indicators: Methodology and user guide París: OECD Publishing.

Saiko, T. A. y Zonn, I. S. (2000). Irrigation expansion and dynamics of desertification in the Circum-Aral region of Central Asia. Applied Geography, 20(4), 349-367. http://dx.doi.org/10.1016/S0143-6228(00)00014-X

Sepehr, A., Hassanli, A. M., Ekhtesasi, M. R. y Jamali, J. B. (2007). Quantitative assessment of desertification in south of Iran using MEDALUS method. Environ Monit Assess, 134(1-3), 243-254.

Sharma, K. D. (1998). The hydrological indicators of desertification. Journal of Arid Environments, 39(2), 121132. http://dx.doi.org/10.1006/jare.1998.0403

Secretaría de Recursos Hidráulicos (SRH). (1971). Regiones hidrológicas núms. 1, 2, 3, 4, 5, 6 y 7. Estado de Baja California y Territorio de Baja California, Boletín Hidrológico, (28).

Secretaría de Salud (México) (SSA). (2001). Norma Oficial Mexicana NOM-127-SSAL-1994. Salud ambiental, agua para uso y consumo humano - límites permisibles de calidad y tratamientos a que debe someterse el agua para su potabilización. México: autor.

Trabelsi, N., Triki, I., Hentati, I. y Zairi, M. (2016). Aquifer vulnerability and seawater intrusion risk using GALDIT, GQI SWI and GIS: case of a coastal aquifer in Tunisia. Environmental Earth Sciences, 75(8).

Valle Junior, R. F., Varandas, S. G. P., Sanches Fernandes, L. F. y Pacheco, F. A. L. (2015). Multi criteria analysis for the monitoring of aquifer vulnerability: a scientific tool in environmental policy. Environmental Science \& Policy, (48), 250-264. http://dx.doi.org/10.1016/j.envsci.2015.01.010

Yohe, G. y Tol, R. (2002). Indicators for social and economic coping capacity-moving toward a working definition of adaptive capacity. Global Environmental Change, (12), 25-40. 